\title{
Controversias en vacunas: seguridad vacunal. ¿Qué sabemos y qué podemos hacer para evitar errores en la práctica diaria?
}

\author{
M. Garcés Sánchez, M. Renales Toboso, P. Minguell González \\ Pediatría. CS Nazaret. Valencia. España.
}

\begin{abstract}
Resumen
Las reacciones adversas o eventos adversos son tan antiguos como los primeros remedios utilizados en el tratamiento de las enfermedades. Las vacunas constituyen, además, un apartado especial ya que se administran a personas sanas para evitar enfermedades y por ello el beneficio obtenido debe ser mayor que el riesgo de presentar una reacción adversa asociada a su administración. Las reacciones adversas siguientes a la vacunación pueden clasificarse en función de su causa. Es muy importante que el personal implicado en la vacunación conozca cuáles son las reacciones adversas que pueden presentarse tras la administración de la vacuna para diferenciarlas de otros eventos inesperados que podrían aparecer, y todas aquellas que puedan estar relacionadas con errores de programa (errores en el almacenamiento, manipulación o administración). A diferencia del resto, estos errores son potencialmente previsibles y, por tanto, evitables. Los eventos acontecidos deben comunicarse al registro de reacciones adversas de los Programas de Farmacovigilancia, donde se evalúa la causalidad del evento y su relación con la vacuna aplicada.
\end{abstract}

Palabras clave: Vacunas. Seguridad vacunal. Farmacovigilancia.

Controversies in vaccines: vaccination security. What do we know and what can we do to avoid errors in the clinical practice?

Abstract

Adverse reactions or adverse events are as old as the first medicines used in treating disease. Besides, vaccines are a special section because they are administered to healthy people to prevent disease and therefore the benefit should be greater than the risk of adverse reactions associated with their administration. Adverse reactions after vaccination can be classified according to their cause. It is very important that staff involved in immunization know what the adverse reactions that may occur after administration of the vaccine are, to differentiate them from other unexpected events that may occur and all those that may be related to program errors (errors in the storage, handling or administration). Unlike the rest, these errors are potentially predictable and therefore preventable. The events should be reported to the Registry of Adverse Reactions' SurveiIlance Programs which assess the causality of the event and its relationship to the given vaccine.

Key words: Vaccines. Vaccine safety. Monitoring immunization safety.

María Garcés Sánchez: maria.garces.sanchez@gmail.com

Los autores declaran no presentar conflictos de intereses en relación con la preparación y publicación de este artículo. 


\section{Introducción}

Las reacciones adversas o eventos adversos son tan antiguos como los primeros remedios utilizados en el tratamiento de las enfermedades. Todo producto con actividad farmacológica puede actuar como tratamiento, pero también como veneno. Ningún producto biológico o farmacéutico desarrollado hasta ahora es $100 \%$ seguro y $100 \%$ eficaz. Pero, ¿qué tipo de eventos adversos podemos encontrarnos asocidados a la práctica vacunal?

Las vacunas constituyen, además, un apartado especial: a diferencia de los demás fármacos, se administran a personas sanas para evitar enfermedades y por ello es necesario que las mismas no causen, como mínimo, más daños de los que podría ocasionar la propia enfermedad; es decir, el beneficio obtenido debe ser mayor que el riesgo de presentar una reacción adversa asociada a su administración. Por ello, deben ser lo más seguras posible.

Todo programa de inmunización tiene como objetivo alcanzar la tasa más elevada de protección frente a la enfermedad inmunoprevenible con el mínimo riesgo de reacciones adversas. Solamente a través de la vigilancia permanente de estos eventos es posible conocer si existe o no causalidad de la vacuna y un episodio adverso acontecido.

\section{Clasificación de las reacciones}

\section{adversas}

Se considera evento adverso (EA) cualquier incidente médico asociado a la administración de una sustancia medicamentosa o droga (por ejemplo, una vacuna) de manera temporal, sin que exista necesariamente una relación causal entre ambos.

Una reacción adversa (RA) medicamentosa es cualquier efecto nocivo, no deseado y no intencional, de una droga, que se presenta cuando la misma se utiliza en dosis usadas normalmente en los seres humanos para la profilaxis, el diagnóstico o el tratamiento de una enfermedad, o para la modificación de una función fisiológica. En este caso, y a diferencia del EA, existe una posibilidad razonable de que haya una relación causal entre el fármaco y la experiencia adversa. Las vacunas, como sustancia medicamentosa de indicación preventiva o terapéutica, están incluidas como causa de ambos conceptos.

Los EA incluyen tanto las RA como los efectos adversos derivados de los errores de administración, efectos nocivos, la intoxicación accidental o intencionada, o el no cumplimiento de la terapia farmacológica.

Por tanto, las RA tras la vacunación pueden considerarse un tipo de EA en el 
que se sospecha una relación causal entre la administración de una vacuna y el efecto observado.

A pesar de estas definiciones y a título práctico, utilizaremos el término reacción adversa para hacer referencia a la clasificación global de las mismas en el contexto de las vacunas, sin establecer diferencias semánticas entre evento y reacción.

Las reacciones adversas siguientes a la vacunación pueden clasificarse, en función de su causa (Organización Mundial de la Salud [OMS]) ${ }^{1}$, en:

- Reacciones inducidas por la vacunación (incluidas reacciones alérgicas).

- Reacciones debidas a errores de programa: errores en el almacenamiento, manipulación o administración.

- Reacciones coincidentes.

- Reacciones idiosincrásicas o de causa desconocida.

\section{Reacciones inducidas por la vacunación}

La finalidad de una vacuna es inducir la inmunidad por medio de la reacción del sistema inmunitario de la persona vacunada. Por tanto, es de esperar que su administración dé lugar a determinados efectos colaterales leves. Estos efectos, que se han estudiados en fase de precomercialización, vienen recogidos en la ficha técnica de los diferentes preparados vacunales.

Una vacuna eficaz y segura reduce estas reacciones al mismo tiempo que induce la inmunidad máxima. Es muy importante que el personal implicado en la vacunación conozca cuáles son las reacciones adversas que pueden presentarse tras la administración de la vacuna y que pueden considerarse como reacciones normales $u$ ordinarias a la misma. A través de este conocimiento pueden detectarse y diferenciarse otros eventos que podrían aparecer: los eventos adversos inesperados. Estos deben ser comunicados al registro de reacciones adversas de los Programas de Farmacovigilancia de cada comunidad autónoma, donde se evalúa la causalidad del evento y su relación con la vacuna aplicada.

Las reacciones inducidas por la vacunación pueden ser locales y sistémicas, y a su vez pueden subclasificarse en comunes, que suelen ser leves, y en raras, que pueden ser más graves. Los efectos secundarios frecuentes suelen ser leves o moderados, sin secuelas permanentes. Rara vez la inmunización produce efectos adversos graves que puedan causar secuelas permanentes o constituyan un peligro para la vida ${ }^{2}$. Los efectos que se producen de manera individual son impredecibles ${ }^{3}$. 
Reacciones debidas a errores

de programa: errores en el

almacenamiento, manipulación

o administración

Las que nos competen en este punto son aquellas que se encuadran fundamentalmente en el grupo de reacciones debidas a errores de programa: errores en el almacenamiento, manipulación o administración. A diferencia del resto, estos errores son potencialmente previsibles $\mathrm{y}$, por tanto, evitables.
Podemos clasificarlas en los siguientes apartados: 1) errores en la prescripción y planificación de la pauta vacunal; 2) errores en el almacenamiento y conservación de las vacunas; 3 ) errores en la manipulación y preparación de las vacunas; y 4) errores inducidos por la técnica vacunal empleada (tabla 1).

\section{Errores en el almacenamiento y conservación de las vacunas}

- Utilización de materiales o productos inmunizantes contaminados o

Tabla 1. Errores de programa y sus consecuencias

\begin{tabular}{|c|c|}
\hline Error de programa & Incidente adverso previsto \\
\hline \multicolumn{2}{|c|}{ Inyección no estéril } \\
\hline $\begin{array}{l}\text { - Reutilización de una jeringa o aguja } \\
\text { desechable } \\
\text { - Esterilización inapropiada de una jeringa } \\
\text { o aguja } \\
\text { - Vacuna o diluyente contaminado } \\
\text { - Reutilización en sesiones posteriores } \\
\text { de la vacuna reconstituida }\end{array}$ & $\begin{array}{l}\text { - Infección, como absceso localizado en el sitio } \\
\text { de la inyección, septicemia, síndrome de } \\
\text { shock tóxico o muerte. Infección transmitida } \\
\text { por la sangre, como hepatitis o VIH }\end{array}$ \\
\hline \multicolumn{2}{|c|}{ Error de reconstitución } \\
\hline $\begin{array}{l}\text { - Reconstrucción con el diluyente incorrecto } \\
\text { - Reemplazo de la vacuna o del diluyente } \\
\text { con un fármaco }\end{array}$ & $\begin{array}{l}\text { - Absceso local por agitación indebida } \\
\text { - Efecto adverso de un fármaco, p. ej., insulina } \\
\text { - Muerte } \\
\text { - Vacuna ineficaz* }\end{array}$ \\
\hline \multicolumn{2}{|c|}{ Inyección en el lugar equivocado: } \\
\hline $\begin{array}{l}\text { - BCG, aplicada por vía subcutánea } \\
\text { - DTP/DT/TT, demasiado superficial } \\
\text { - Inyección en la nalga }\end{array}$ & $\begin{array}{l}\text { - Reacción o absceso local } \\
\text { - Reacción o absceso local } \\
\text { - Daño al nervio ciático }\end{array}$ \\
\hline Caso omiso de las contraindicaciones & Reacción grave prevenible \\
\hline
\end{tabular}


en condiciones de conservación inadecuadas. Puede provocar los siguientes accidentes adversos previstos: infección local, en forma de abceso localizado en el lugar de punción; infección generalizada, en forma de septicemia, shock tóxico y muerte; infección transmitida por vía hematológica, como hepatitis o VIH.

- Transporte o almacenamiento incorrecto. Puede provocar: reacción local por vacuna congelada, vacunación ineficaz por pérdida de la actividad del producto vacunal.

\section{Errores en la manipulación}

\section{y preparación de las vacunas}

- Errores de reconstrucción de la vacuna con el diluyente o reemplazo de la misma. Puede provocar: absceso local por la agitación indebi$\mathrm{da}$, el efecto adverso asociado al producto administrado o incluso la muerte.

\section{Errores inducidos por la técnica vacunal empleada}

- Inyección en el lugar equivocado, administración incorrecta en el lugar aconsejado, o administración diferente a la vía aconsejada por el fabricante. Puede provocar: Reacciones o abscesos locales, lesión del nervio ciático ${ }^{4,5}$.

\section{Reacciones coincidentes}

Se producen cuando la causa de la reacción adversa es coincidente con el acto vacunal. Ello significa que el accidente médico pudo haber ocurrido aunque el paciente no hubiera sido vacunado. La mejor evidencia para concluir que un evento adverso es coincidente, es que el mismo haya sido diagnosticado en personas que no han sido vacunadas.

\section{Reacciones idiosincrásicas}

\section{o de causa desconocida}

Son reacciones que aparecen en un paciente tras la vacunación, cuya causa no corresponde a ninguna de las tres mencionadas anteriormente $y$, por lo tanto, se desconoce la causa que las produce.

\section{Actitud y profilaxis frente a las reacciones adversas vacunales}

Los profesionales sanitarios implicados en el acto vacunal deben conocer las reacciones adversas más frecuentes para poder identificarlas de forma precoz y adoptar las medidas oportunas lo más rápidamente posible. 
Tres son las funciones básicas ${ }^{6,7}$ que deben desarrollarse desde la Atención Primaria de salud del niño en la vigilancia de los efectos adversos de las vacunas:

- Función educativa, explicando a los padres y tutores los beneficios que se derivan de la vacunación, las enfermedades que previenen, alertando de los posibles efectos secundarios que pueden aparecer.

- Función preventiva, conociendo las reacciones adversas más frecuentes para su correcta identificación y tratamiento, conociendo las contraindicaciones verdaderas para evitar posponer innecesariamente las inmunizaciones indicadas e identificar los pacientes con mayor riesgo de reacciones adversas o graves. Así mismo, deben tener la formación adecuada para la identificación de reacciones anafilácticas y urgencias asociadas a la administración de vacunas potencialmente tratables $y$ para el manejo pertinente de las mismas.

- Función de vigilancia, activa o pasiva, detectando eventos adversos inesperados, comunicándolos a través de los programas de farmacovigilancia.
Función educativa: información sobre seguridad de vacunas

Los riesgos derivados del acto vacunal, aunque escasos y, en su mayoría, leves, pueden aparecer de forma impredecible. Por ello, los padres o tutores del paciente que recibe la vacuna deben conocer, además de los beneficios que puede aportar la inmunización activa a sus hijos, los efectos adversos que pueden aparecer tras la administración de la misma. Es necesario que disipen sus dudas o temores y se les debe alentar a preguntar todo aquello que necesiten para sentirse bien informados. Previo a la administración es necesario interrogar a los padres sobre el paciente para descartar la existencia de algún factor que contraindique la vacunación.

Los padres deben saber que:

- Los riesgos de contraer una enfermedad grave por no administrar la vacuna son mucho mayores que el riesgo de que la vacuna produzca una reacción adversa importante.

- Las vacunas pueden tener efectos secundarios, que varían en intensidad según el tipo de vacuna que se administre. Pueden ser locales (enrojecimiento, hinchazón o dolor en el lugar de la punción) o sistémicos (fiebre leve). Hay que advertirles que es normal que exista 
una ligera molestia durante varios días, que no debe ser motivo de alarma.

- Deben consultarnos ante cualquier reacción importante o persistente que presente el paciente, para que podamos proceder a evaluarla y valoremos si puede existir relación con el acto vacunal.

\section{Función preventiva: formación} adecuada sobre las reacciones previsibles frecuentes y capacidad de actuación sobre las inusuales

Las reacciones inducidas por la vacunación son, por lo general, difíciles o imposibles de prevenir por el personal implicado en el acto vacunal. El conocimiento de la existencia y de la frecuencia de aparición de los efectos adversos de cada vacuna por parte del profesional sanitario junto con el despistaje de la existencia de factores que la contraindiquen o nos exijan tomar precauciones, puede contribuir a disminuir el riesgo.

A diferencia de las reacciones inducidas por la vacunación, las reacciones debidas a errores del programa pueden limitarse o prevenirse en gran medida, dado que en su mayoría son producidas por errores humanos. Puede prevenirse mediante la capacitación debida del personal responsable de la vacunación, facilitándole su formación continuada, y el suministro apropiado y seguro del equipo de inyección.

Las reglas básicas para evitar los errores de programa son (OMS):

- Utilizar aguja y jeringuilla estériles para cada inyección.

- Reconstituir la vacuna únicamente con el diluyente proporcionado por el fabricante para la vacuna.

- Desechar la vacuna reconstituida después de seis horas y nunca conservarla durante toda la noche.

- Utilizar un refrigerador específico para el almacenamiento de las vacunas, ya que estas deben permanecer a temperatura constante $y$ reiteradas aperturas del refrigerador podrían alterar sus cualidades, usando uno diferente para almacenar los fármacos y otras sustancias.

- Capacitar y supervisar adecuadamente al personal implicado para que observen las prácticas seguras de inyección.

- Investigar cualquier error de programa para que este no se repita.

\section{Función de vigilancia: registro de vacunas, farmacovigilancia, notificación de reacciones adversas \\ El registro de vacunación constituye una de las fuentes de datos primarios}


del sistema de información sobre inmunización. Su función es conocer el estado vacunal del paciente, permitiendo evaluar correctamente las vacunaciones y así reducir los casos de calendarios vacunales incompletos. Así mismo, sirve como historia vacunal del paciente y permite transmitir información entre los diferentes profesionales sanitarios?

La farmacovigilancia tiene como objeto la detección, verificación y cuantificación de las reacciones adversas a los medicamentos, minimizando así los riesgos derivados de la administración de un producto farmacológico. Esto permite establecer medidas reguladoras en caso necesario, además de ofrecer información a los profesionales sanitarios y al público en general sobre la naturaleza de los eventos detectados tanto en las fases de desarrollo clínico del producto como durante la fase poscomercialización ${ }^{8}$.

El Sistema Español de Farmacovigilancia (SEFV), coordinado por el Ministerio de Sanidad y Consumo, es el órgano interterritorial integrado por administraciones sanitarias autonómicas, por Centros Regionales de Farmacovigilancia (CRFV) y por la División de Farmacovigilancia y Farmacoepidemiología de la Agencia del Medicamento, conectado con el programa oficial por la Organización Mundial de la Salud. Su función es recopilar y elaborar la información sobre reacciones adversas medicamentosas.

Los profesionales sanitarios deben comunicar los efectos adversos que podrían estar en relación con la administración de un medicamento. Para ello disponen del sistema de Notificación Espontánea de Reacciones Adversas, o "Tarjeta Amarilla", que consiste en un formulario elaborado para recoger las notificaciones de sospecha de reacciones adversas. Se basa en la notificación voluntaria, por parte de los profesionales sanitarios, de cualquier sospecha de Reacción Adversa observada en relación con el empleo de los medicamentos. En dicho formulario se recoge toda la información necesaria para analizar cada sospecha de reacción adversa. Se solicitan los siguientes datos:

- Nombre del paciente: para identificar repetidas reacciones adversas en un mismo sujeto.

- Edad: para identificar grupos de riesgo.

- Peso: para valorar sobredosificación.

- Vacuna (nombre comercial y número de lote): para identificar reacciones secundarias al excipiente $y / 0$ forma farmacéutica. 
- Dosis diaria y vía de administración: para establecer relación temporal de la reacción adversa en una politerapia y detectar errores en la técnica.

- Comienzo y final de la administración: para valorar cronológicamente la exposición y detectar manifestación, descartando patología causal alternativa.

- Motivo de la prescripción: para valorar posible relación con patología de base.

- Descripción detallada de la reacción adversa.

- Desenlace: para detectar la gravedad.

- Observaciones adicionales clínicas o analíticas: ayudan a precisar la imputabilidad (relación causa-efecto) a la vacuna.
- El médico que notifica, requiere que se le facilite la retroinformación sobre el fenómeno y el seguimiento.

Para las vacunas de reciente comercialización deben comunicarse todas las sospechas de reacciones adversas, independientemente de la gravedad ${ }^{9}$. La definición de medicamentos de reciente comercialización es un tanto arbitraria, se acepta un periodo de tres a cinco años, pero es variable en función de la frecuencia de uso de ese medicamento.

Para todos los medicamentos hay que comunicar: reacciones graves, es decir, que determinen el fallecimiento o riesgo de fallecimiento del sujeto, incapacidad permanente o significativa, y hospitalización o prolongación de esta.

\section{Bibliografía}

1. WHO/V\&B/00.36. Supplementary information on vaccine safety. Part 2: Background rates of adverse events following immunization.

2. Bohlke K, Davis RL, Marcy SM, Braun MM, DeStefano F, Black SB et al; Vaccine Safety Datalink Team. Risk of anaphylaxis after vaccination of children and adolescents. Pediatrics. 2003;112:815-20.

3. Díez Domingo J, Planelles MV, Baldó JM, Ballester $A$, Núñez $F$, Jubert $A$ et al. Randomized trial of prophylaxis vs treatment of adverse reactions to DTP vaccination with ibuprofen. Curr Ther Res. 1998;59:579-88.

4. Piyasirisilp S, Hemachudha T. Neurological adverse events associated with vaccination. Curr Opin Neurol. 2002;15:333-8.

5. Eseverri JL, Ranea S, Marin A. Reacciones adversas a las vacunas. Allergol Immunopathol. 2003;31:125-38.

6. Picazo J. Reacciones adversas. En: Guía práctica de vacunaciones. Madrid: Centro de estudios de Ciencias de la Salud; 2002. 
7. Comité Asesor de Vacunas de la Asociación Española de Pediatría. Reacciones adversas de las vacunas. En: Manual de Vacunas de la AEP, edición 2001. Madrid: AEP; 2001. p. 507-37.

8. Mollet J, MacDonald M, Cashman N, Pless R. Vigilancia de la seguridad de las vacunas: evaluación de las notificaciones de episodios adver- sos por un comité consultivo de expertos. Bull WHO. 2000;78:178-85.

9. CDC. Update: Vaccine side effects, adverse reactions, contraindications and precaution. Recommendations of the Advisory Committee on Immunization Practices. MMWR. 1996;45(RR12):1-35. 